\title{
Long-range correlations improve understanding of the influence of network structure on contact dynamics
}

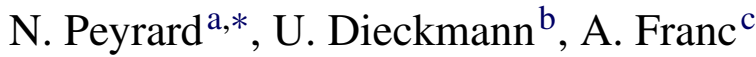 \\ ${ }^{a}$ Unité de Biométrie et Intelligence Artificielle, UR 875, INRA, Toulouse, France \\ ${ }^{\mathrm{b}}$ Evolution and Ecology Program, International Institute for Applied Systems Analysis, A-2361 Laxenburg, Austria \\ c INRA, UMR 1202 Biodiversité, Gènes et Communautés, Bordeaux, France
}

Received 18 December 2006

Available online 11 February 2008

\begin{abstract}
Models of infectious diseases are characterized by a phase transition between extinction and persistence. A challenge in contemporary epidemiology is to understand how the geometry of a host's interaction network influences disease dynamics close to the critical point of such a transition. Here we address this challenge with the help of moment closures. Traditional moment closures, however, do not provide satisfactory predictions close to such critical points. We therefore introduce a new method for incorporating longer-range correlations into existing closures. Our method is technically simple, remains computationally tractable and significantly improves the approximation's performance. Our extended closures thus provide an innovative tool for quantifying the influence of interaction networks on spatially or socially structured disease dynamics. In particular, we examine the effects of a network's clustering coefficient, as well as of new geometrical measures, such as a network's square clustering coefficients. We compare the relative performance of different closures from the literature, with or without our long-range extension. In this way, we demonstrate that the normalized version of the Bethe approximation - extended to incorporate long-range correlations according to our method - is an especially good candidate for studying influences of network structure. Our numerical results highlight the importance of the clustering coefficient and the square clustering coefficient for predicting disease dynamics at low and intermediate values of transmission rate, and demonstrate the significance of path redundancy for disease persistence.
\end{abstract}

(C) 2007 Elsevier Inc. All rights reserved.

Keywords: Contact process; Interaction-network structure; Long-range correlation; Moment closure; Phase transition

\section{Introduction}

Infectious diseases without permanent immunity are classically represented as SIS (susceptible-infected-susceptible) systems. Such models can be implemented on lattices, or more generally on graphs. The resultant models are characterized by a dichotomy between two possible dynamics: the disease either undergoes rapid extinction or it persists over a long time. This change of behaviour can be understood as a bifurcation or phase transition governed by properties such as a disease's transmission rate. The value of the control parameter at which such a transition occurs is known as the critical value. Critical values have received considerable attention in statistical physics because (i) they delimit ranges of parameters values within which

* Corresponding address: Unité BIAT, INRA, Chemin de Borde-Rouge, BP 52627, 31326 Castanet-Tolosan cedex, France.

E-mail address: nathalie.peyrard@toulouse.inra.fr (N. Peyrard). the qualitative behaviour of a system remains unchanged and (ii) classical approximations - such as the mean-field approximation - are known to yield accurate predictions only far way from critical values. Studying the critical dynamics of an infectious disease is important for understanding the outbreaks and prevalence of the disease close to the transition. A better knowledge of factors influencing critical values is therefore important for improving disease control.

In many spatially or socially structured disease models, the transmission is restricted to nearest neighbours. The geometrical pattern of transmission-enabling contacts between hosts can be described by a graph. The resultant interaction networks play an important role in the understanding and prediction of disease dynamics (Keeling, 1999; Eguiluz and Klemm, 2002; Keeling, 2005; Colizza et al., 2006). In particular, the geometrical features of such networks can influence the critical values of a disease's phase transition. 
However, while the existence of this influence is well known, the important geometrical characteristics of arbitrary networks, favouring or limiting disease propagation and persistence, are still not understood in general. For a long time, the study of interaction networks has been restricted to random graphs (resulting in mean-field models when fully connected, or in island models otherwise) or to regular lattices (resulting, e.g. in stepping-stone models). More realistic families of graphs have received mounting attention only recently: these graphs are characterized by statistical features such as degree distributions and clustering coefficients (Albert and Barabási, 2002; Colizza et al., 2006). In this study, we propose new geometric characteristics of graphs that are particularly useful for characterizing disease dynamics.

Individual-based spatially explicit stochastic systems are often called interacting particle systems (IPSs; Liggett, 1985; Durrett and Levin, 1994). IPSs are useful for capturing the propagation of infectious diseases (e.g. Keeling et al., 2003). They are also often used to describe ecological dynamics, especially when individual contacts occur among nearest neighbours (Van Baalen and Rand, 1998). However, very few exact results have been demonstrated for IPS models, which, therefore, are mostly studied through simulations. By applying mean-field approximations, IPSs can be reduced to ODE (ordinary differential equation) models, with suitable state variables, such as the mean densities of susceptible and infected hosts. Critical values can then be approximated by the bifurcation points of these ODE models. However, examples of the failure of such mean-field models close to critical values are numerous (Durrett and Levin, 1994; Filipe and Gibson, 1998; Dieckmann et al., 2000, Part B; Keeling, 2005). A common reason for these failures is the inability of mean-field models to capture correlations in the state of neighbouring individuals. Therefore, considerable attention has been given to the design of approximations that behave better than mean-field approximations in the vicinity of the critical values of an IPS model. Such methods are often referred to as moment closure methods (Bolker and Pacala, 1997). Prominent examples include the pair approximation (Matsuda et al., 1992) and cluster variation methods (Kikuchi, 1951; Krishnarajah et al., 2005) such as the Bethe approximation. While these methods have become popular in theoretical epidemiology and ecology (e.g. Dieckmann et al., 2000), they account for a network's structure only in limited ways, and thus cannot be used to study the influence of such structure more in general.

Analytically tractable models of statistical physics, such as the Ising model, reveal that approximations often break down because of the persistence, or even amplification, of long-range correlations close to critical values (Marro and Dickman, 1999; Snyder and Nisbet, 2000; Dickman and Martins de Oliveira, 2005). Hence, one way to improve upon classical approximations is to take long-range correlations into account, to the extent possible, thereby going beyond the correlations between nearest neighbours, which are the basis of the classical pair approximations and Bethe approximations on graphs. Here we take as our point of departure several published approximations relying on correlations between nearest neighbours only, and propose for each of them new approximations by incorporating correlations at distances larger than 1. We show that, in some cases, this method leads to more accurate predictions of critical parameter values, and also better describes the prevalence of a disease. In addition, our new approximations allow us to explicitly take into account geometrical features of graphs not captured by the classical approximations, which makes it possible to study their influence on disease prevalence.

The new method introduced here is but one of several propositions for improving classical approximations (Sato et al., 1994; Snyder and Nisbet, 2000; Filipe and Gibson, 2001; Petermann and De Los Rios, 2004a). None of these earlier methods, however, was dedicated to exploring and quantifying the influence of the distinctive geometric features graphs possess. Working in this direction, Petermann and De Los Rios (2004b) suggested an alternative method for improvements, through the careful examination of basic clusters defined by local patterns larger than pairs. While the resultant approximations can improve the description of disease dynamics for a given interaction network, the systematic comparison of disease dynamics across different types of graph is not straightforward, since different basic clusters would then have to be identified and used as the basis of (hence essentially different) approximation schemes.

To illustrate our approach, we focus on a simple model of disease propagation on graphs by analysing the well-studied contact process (Harris, 1974; Marro and Dickman, 1999), which serves as a standard for the spatially explicit definition of SIS models. This model has been used for modeling phenomena involving excitable media, like forest-fire models (Drossel and Schwabl, 1992), or for modelling the ecological dynamics of metapopulations (Franc, 2004). The contact process has also been widely used for studying the spread of diseases (Filipe and Gibson, 1998; Pastor-Satorras and Vespignani, 2001; Eguiluz and Klemm, 2002).

This article is organized as follows. Section 2 introduces the contact process, and Section 3 reviews classical moment closures. In Section 4, we explain in detail, using the Bethe approximation as an example, the main methodological contribution of this study: how to extend moment closure approximations based on nearest neighbours to longer-range correlations. Exploiting the insights thus obtained, we propose new geometrical characteristics of graphs that have the potential to influence the dynamics of contact processes. In Section 5, we present a comparison of different approximation schemes, by critically evaluating approximations that have already been published against approximations based on our new method. We select the approximation best suited for studying the influence of the identified graph characteristics. We conclude with a discussion of possible improvements of our method and with guidelines for selecting suitable moment closures

\section{The contact process}

The contact process is a simple model for describing the spread of an epidemic in continuous time (Harris, 1974; 
Marro and Dickman, 1999). We consider a graph, whose nodes are individual hosts and whose edges connect hosts that can infect one another. We will focus on graphs with homogeneous degree, in which each node has the same number $h$ of neighbours. At any time $t>0$, a host $i$ can be in either of two states $z_{i}$ : susceptible $\left(z_{i}=0\right)$ or infected $\left(z_{i}=1\right)$. If $i$ is infected, it becomes susceptible with probabilistic rate $\mu$. As units are arbitrary, we choose $\mu=1$ without loss of generality, so that the unit of time is the expected duration between a host's infection and recovery. If $i$ is susceptible and has $a_{i}$ infected neighbours, it becomes infected with probabilistic rate $\beta a_{i}$, with $\beta$ denoting the probabilistic transmission rate between neighbouring hosts,

$P\left(z_{i}^{t+d t}=0 \mid z_{i}^{t}=1\right)=d t$,

$P\left(z_{i}^{t+d t}=1 \mid z_{i}^{t}=0, a_{i}=a\right)=a \beta d t$.

This process exhibits a critical value of the transmission rate that depends on the considered graph: for a transmission rate below the critical value the infection dies out, while above this value the infection spreads.

There is no known analytical solution to the contact process, and several approximations have been derived. Approximations based on moment closure have been shown to behave fairly well far from the critical point; these are described in the next section. Our goal in this study is to propose an improvement of existing moment closure approximations close to the critical point by taking into account correlations between hosts at distances larger than that between nearest neighbours.

\section{Moment dynamics and moment closures}

Instead of tracing through time the states of all nodes of a graph, the essential features of the contact process dynamics can be captured by salient summary statistics. ODEs can thus be constructed to describe the expected dynamics of these statistics over realizations of the stochastic process. Note that in the following, for the sake of simplicity we will adopt the same notations for empirical frequencies and expected quantities (probabilities).

\subsection{Singlet dynamics}

We denote the probability that a host is infected at time $t$ by $\rho(t)$. The dynamics of $\rho$ are then given by

$\frac{d \rho}{d t}=h \beta P^{1}(01)-\rho$,

(Filipe and Gibson, 1998; Peyrard and Franc, 2005), where $P^{1}(01)$ is the probability that a pair of nodes linked by an edge is in state 01. Such nodes are also said to be (nearest) neighbours, adjacent, or directly connected; their distance is defined as 1 . More generally, here and in the following $P^{d_{i j}}\left(z_{i}, z_{j}\right)$ denotes the probability for two arbitrary nodes $i$ and $j$ at distance $d_{i j}$ to be in states $z_{i}$ and $z_{j}$. The symmetry relation $P^{d_{i j}}\left(z_{i}, z_{j}\right)=P^{d_{i j}}\left(z_{j}, z_{i}\right)$ holds by definition. These probabilities do not refer to specific nodes and must be interpreted as expectations over all pairs in the graph that possess the specified distance.

\subsection{Pair dynamics}

Closing Eq. (2) requires information about the dynamics of $P^{1}(01)$,

$$
\begin{aligned}
\frac{d P^{1}(01)}{d t}= & P^{1}(11)+\beta(h-1) P^{1,1}(100) \\
& -P^{1}(01)\left[1+\beta+\beta(h-1) \frac{P^{1,1}(101)}{P^{1}(01)}\right]
\end{aligned}
$$

(Filipe and Gibson, 1998; Peyrard and Franc, 2005), where $P^{1,1}(100)$ is the probability to observe a node in state 0 and two of its neighbours in states 0 and 1 , respectively. The definition of $P^{1,1}(101)$ is analogous. Denoting the set of all neighbouring pairs of nodes by $E$, we thus have $P^{1,1}(100)=P\left(z_{i}=\right.$ $\left.1, z_{j}=0, z_{k}=0 \mid(i, j),(j, k) \in E\right)$ and $P^{1,1}(101)=$ $P\left(z_{i}=1, z_{j}=0, z_{k}=1 \mid(i, j),(j, k) \in E\right)$. Eq. (3) may seem complicated at first sight, but is nothing more than a straightforward consequence of accounting for infection and recovery events starting from, or leading to, a pair of neighbours in state 01 (Appendix A.1 provides details on this derivation). Note that the roles of nodes $i$ and $k$ in the triplets discussed here are equivalent, while that of the central node $j$ is different: it is for this reason that the ordering of states in $P^{1,1}(100)=P^{1,1}(001)$ and $P^{1,1}(101)$ is important. It also needs to be appreciated that the triplets $(i, j, k)$ can either be open or closed (resulting in a triangular configuration of edges), depending on whether or not nodes $i$ and $k$ are neighbours.

Just as the dynamics of singlets in Eq. (2) depend on pair states, the dynamics of pairs in Eq. (3) depend on triplet states. In general, whenever interactions occur only along edges, the dynamics of configurations of order $n$ depend on the state of configurations of order $n+1$. To close a dynamic system like the one jointly given by Eqs. (2) and (3), one therefore needs to truncate the resultant hierarchy of equations at a certain order by approximating the required higher-order joint probabilities as functions of lower-order ones. Such relations are referred to as moment closures. Moment closure approximations go back to the cluster variation method developed in solid-state physics during the 1930s (Kikuchi, 1951; Lavis and Bell, 1999).

\subsection{Order-1 closure}

The simplest closure is a closure at order 1, also known as the mean-field approximation $(M F): P^{1}(01)=\rho(1-\rho)$. This yields a closed equation in $\rho$ (Marro and Dickman, 1999),

$\frac{d \rho}{d t}=h \beta \rho(1-\rho)-\rho$.

The SIS model often used in non-spatial epidemiology is thus recovered (with the probability $\rho$ being interpreted as the proportion, or frequency, of infected hosts). Eq. (4) is easy to solve and study, but except in particular cases (for contact graphs with a high value of $h$, for contact graphs in high dimensions, or for diseases with high prevalence) the meanfield assumption, implying a well-mixed population of hosts, is too restrictive, so that this approximation's performance typically is quantitatively poor (Durrett and Levin, 1994; Filipe and Gibson, 1998; Dieckmann et al., 2000; Keeling, 2005). 


\subsection{Order-2 closures}

In order-2 closures, triplet probabilities are approximated in terms of singlet and pair probabilities. Correlations between two nodes linked by an edge are no longer ignored. However, two nodes at distance larger than 1 are still assumed to be independent. Let us introduce here the notation for triplet probabilities: $P^{d_{i j}, d_{j k}, d_{i k}}\left(z_{i}, z_{j}, z_{k}\right)$ and $\widehat{P}^{d_{i j}, d_{j k}, d_{i k}}\left(z_{i}, z_{j}, z_{k}\right)$ denote the exact and approximated probabilities, respectively, for three arbitrary nodes $i, j$, and $k$ at distances $d_{i j}, d_{j k}$, and $d_{i k}$ to be in states $z_{i}, z_{j}$, and $z_{k}$. As for pair probabilities, these triplet probabilities must be interpreted as expectations over all triplets in the graph that possess the specified distances. Corresponding probabilities that are applicable when the third distance $d_{i k}$ is unknown are denoted analogously, by $P^{d_{i j}, d_{j k}}\left(z_{i}, z_{j}, z_{k}\right)$ and $\widehat{P}^{d_{i j}, d_{j k}}\left(z_{i}, z_{j}, z_{k}\right)$. For an example see Eq. (6b).

The most classical order-2 closure is known as pair approximation $(P A)$. This approximation (Matsuda et al., 1992) has become a standard for simplifying spatiotemporal complexity in epidemiological and ecological models in which space is discrete (Iwasa, 2000; Sato and Iwasa, 2000; Van Baalen, 2000),

$$
\begin{aligned}
\widehat{P}_{P A}^{1,1,1}\left(z_{i}, z_{j}, z_{k}\right) & =\widehat{P}_{P A}^{1,1,2}\left(z_{i}, z_{j}, z_{k}\right)=\widehat{P}_{P A}^{1,1}\left(z_{i}, z_{j}, z_{k}\right) \\
& =\frac{P^{1}\left(z_{i}, z_{j}\right) P^{1}\left(z_{j}, z_{k}\right)}{P\left(z_{j}\right)} .
\end{aligned}
$$

Note that $\widehat{P}_{P A}^{1,1, d_{i k}}\left(z_{i}, z_{j}, z_{k}\right)=0$ for $d_{i k}$ larger than 2 since the third distance is always less or equal to 2 . The pair approximation implies the assumption that the states of nodes $i$ and $k$ are independent given the state of node $j$.

This limitation can be overcome by the so-called Bethe approximation $(B A)$, which distinguishes between closed triplets $\left(d_{i k}=1\right)$ and open triplets $\left(d_{i k}=2\right)$. Denoting by $\theta$ the graph's clustering coefficient (i.e. the probability that two neighbours of a node are neighbours of each other; Albert and Barabási, 2002; Newman, 2003), $P^{1,1}$ can, in general, be expressed as $P^{1,1}=\theta P^{1,1,1}+(1-\theta) P^{1,1,2}$. The Bethe approximation (Morita, 1994; Yedidia et al., 2005) for closed triplets is then given by the so-called Kirkwood approximation (Singer, 2004),

$\widehat{P}_{B A}^{1,1,1}\left(z_{i}, z_{j}, z_{k}\right)=\frac{P^{1}\left(z_{i}, z_{j}\right) P^{1}\left(z_{j}, z_{k}\right) P^{1}\left(z_{i}, z_{k}\right)}{P\left(z_{i}\right) P\left(z_{j}\right) P\left(z_{k}\right)}$,

while the Bethe approximation for open triplets is given by the pair approximation, $\widehat{P}_{B A}^{1,1,2}=\widehat{P}_{P A}^{1,1,2}$. Accordingly, the Bethe approximation for $P^{1,1}$ is defined by

$$
\begin{aligned}
\widehat{P}_{B A}^{1,1}\left(z_{i}, z_{j}, z_{k}\right)= & \theta \widehat{P}_{B A}^{1,1,1}\left(z_{i}, z_{j}, z_{k}\right) \\
& +(1-\theta) \widehat{P}_{B A}^{1,1,2}\left(z_{i}, z_{j}, z_{k}\right) .
\end{aligned}
$$

However, as opposed to pair approximation, the Bethe approximation of closed-triplet probabilities according to Eq. (6a) is not normalized, so that the probabilities $\widehat{P}_{B A}^{1,1,1}\left(z_{i}, z_{j}, z_{k}\right)$ when summed over all combinations of $z_{i}, z_{j}$, and $z_{k}$ do not necessarily add up to 1 . We will therefore also consider the following, appropriately normalized version of the
Bethe approximation $(N B A)$,

$$
\widehat{P}_{N B A}^{1,1,1}\left(z_{i}, z_{j}, z_{k}\right)=\frac{\widehat{P}_{B A}^{1,1,1}\left(z_{i}, z_{j}, z_{k}\right)}{\sum_{z_{i}, z_{j}, z_{k}} \widehat{P}_{B A}^{1,1,1}\left(z_{i}, z_{j}, z_{k}\right)} .
$$

Moment closures have been used to approximate population dynamics in continuous space. In this context, other order-2 closures have been proposed recently (Law and Dieckmann, 2000; Dieckmann and Law, 2000; Law et al., 2003; Murrel et al., 2004). They are referred to as power-1 closure $(P 1)$ and power-2 closure $(P 2)$ according to the number of pair probabilities that get multiplied in the terms of the closure,

$$
\begin{aligned}
& \widehat{P}_{P 1}^{1,1,1}\left(z_{i}, z_{j}, z_{k}\right) \\
& \quad=P\left(z_{i}\right) P^{1}\left(z_{j}, z_{k}\right)+P\left(z_{j}\right) P^{1}\left(z_{i}, z_{k}\right)+P\left(z_{k}\right) P^{1}\left(z_{i}, z_{j}\right) \\
& \quad-2 P\left(z_{i}\right) P\left(z_{j}\right) P\left(z_{k}\right) \\
& \widehat{P}_{P 2}^{1,1,1}\left(z_{i}, z_{j}, z_{k}\right) \\
& \quad=\frac{1}{2}\left[\frac{P^{1}\left(z_{i}, z_{j}\right) P^{1}\left(z_{j}, z_{k}\right)}{P\left(z_{j}\right)}+\frac{P^{1}\left(z_{i}, z_{j}\right) P^{1}\left(z_{i}, z_{k}\right)}{P\left(z_{i}\right)}\right. \\
& \left.\quad+\frac{P^{1}\left(z_{i}, z_{k}\right) P^{1}\left(z_{j}, z_{k}\right)}{P\left(z_{k}\right)}-P\left(z_{i}\right) P\left(z_{j}\right) P\left(z_{k}\right)\right]
\end{aligned}
$$

The corresponding equations for $\widehat{P}^{1,1,2}$ are obtained by replacing $P^{1}\left(z_{j}, z_{k}\right)$ with $P\left(z_{i}\right) P\left(z_{k}\right)$. For obvious reasons, the Bethe/Kirkwood approximation is referred to as a power3 closure (Law and Dieckmann, 2000; Dieckmann and Law, 2000; Law et al., 2003; Murrel et al., 2004). A drawback of the power- 1 and power- 2 closures is that they may lead to negative values (which then have to be replaced with 0 ). For the contact process, this may happen when $\beta$, and thus $\rho$, are low.

\section{Extensions to longer-range correlations}

For each of the five closures described above $(P A, B A$, $N B A, P 1$, and $P 2$ ), it is implicitly assumed that any two nodes at distances larger than 1 are independent. We will now show how to surmount this restriction, by deriving extended versions of these closures that account for correlations at larger distances. A method for incorporating distance- 2 and distance3 correlations is explicitly detailed and illustrated for the Bethe closure. While the presented method readily extends to larger distances, only short correlation distances are computationally tractable.

\subsection{Incorporating distance-2 correlations}

We define the extended Bethe approximation as

$$
\begin{aligned}
\widehat{P}^{d_{i j}, d_{j k}, d_{i k}}\left(z_{i}, z_{j}, z_{k}\right) \\
=\frac{P^{d_{i j}}\left(z_{i}, z_{j}\right) P^{d_{j k}}\left(z_{j}, z_{k}\right) P^{d_{i k}}\left(z_{i}, z_{k}\right)}{P\left(z_{i}\right) P\left(z_{j}\right) P\left(z_{k}\right)} .
\end{aligned}
$$

In the above expression, we approximate a pair probability, for instance $P^{d_{i k}}\left(z_{i}, z_{k}\right)$, with $P\left(z_{i}\right) P\left(z_{k}\right)$ if $d_{i k}>2$ (instead of if $d_{i k}>1$, as assumed in the classical closures). 
To construct an extended Bethe approximation, we again use the relationship $P^{1,1}=\theta P^{1,1,1}+(1-\theta) P^{1,1,2}$. The approximation of $P^{1,1,1}\left(z_{i}, z_{j}, z_{k}\right)$ applies to closed triplets and remains given by Eq. (6a). For $P^{1,1,2}\left(z_{i}, z_{j}, z_{k}\right)$, i.e. for an open triplet, even if there is no edge between nodes $i$ and $k$, many other paths of lengths larger than 1 may exist. The shortest of them is the path through the central node $j$ of the triplet, which is a path of length 2 . Taking into account this correlation (which of course is only part of the set of the long-range correlations existing along all possible paths), the distance-2 Bethe closure for $P^{1,1,2}\left(z_{i}, z_{j}, z_{k}\right)$ is

$\widehat{P}^{1,1,2}\left(z_{i}, z_{j}, z_{k}\right)=\frac{P^{1}\left(z_{i}, z_{j}\right) P^{1}\left(z_{j}, z_{k}\right) P^{2}\left(z_{i}, z_{k}\right)}{P\left(z_{i}\right) P\left(z_{j}\right) P\left(z_{k}\right)}$.

The correction factor relative to the classical Bethe approximation is $\frac{P^{2}\left(z_{i}, z_{k}\right)}{P\left(z_{i}\right) P\left(z_{k}\right)}$ and accounts for the fact that the states $z_{i}$ and $z_{k}$ are not independent.

\subsection{Resultant closed dynamic system}

We can now exploit the distance- 2 order- 2 closure defined above to close the open dynamic system given by Eqs. (2) and (3). This will be achieved by approximating $\widehat{P}^{1,1}(100)$ based on Eqs. (6a) and (11),

$$
\begin{aligned}
& \widehat{P}^{1,1}(100)=\theta \widehat{P}^{1,1,1}(100)+(1-\theta) \widehat{P}^{1,1,2}(100) \\
& =\theta \frac{P^{1}(01)^{2} P^{1}(00)}{\rho(1-\rho)^{2}}+(1-\theta) \frac{P^{1}(01) P^{1}(00) P^{2}(01)}{\rho(1-\rho)^{2}} \\
& =\frac{P^{1}(01) P^{1}(00)}{\rho(1-\rho)^{2}}\left[\theta P^{1}(01)+(1-\theta) P^{2}(01)\right] .
\end{aligned}
$$

The approximation $\widehat{P}^{1,1}(101)$ is defined analogously based on a decomposition with respect to the third distance. We thus see that an additional equation is required for describing the dynamics of $P^{2}(01)$. Denoting by $T^{2}\left(z_{1}, z_{2} \rightarrow z_{3}, z_{4}\right)$ the transition rate of two nodes at distance 2 from states $z_{1}$ and $z_{2}$ to states $z_{3}$ and $z_{4}$, respectively, and accounting for all four types of transition starting from or leading to state 01 , we obtain

$$
\begin{aligned}
& \frac{d P^{2}(01)}{d t}=-P^{2}(01)\left[T^{2}(01 \rightarrow 11)+T^{2}(01 \rightarrow 00)\right] \\
& \quad+P^{2}(00) T^{2}(00 \rightarrow 01)+P^{2}(11) T^{2}(11 \rightarrow 01)
\end{aligned}
$$

The involved transition rates at distance 2 are then approximated by

$$
\begin{aligned}
& T^{2}(01 \rightarrow 11)=\beta(h-1) \frac{\widehat{P}^{1,2}(101)}{P^{2}(01)}+\beta \frac{\widehat{P}^{1,1,2}(011)}{P^{2}(01)}, \\
& T^{2}(01 \rightarrow 00)=1, \\
& T^{2}(00 \rightarrow 01)=\beta(h-1) \frac{\widehat{P}^{1,2}(100)}{P^{2}(00)}+\beta \frac{\widehat{P}^{1,1,2}(010)}{P^{2}(00)}, \\
& T^{2}(11 \rightarrow 01)=1 .
\end{aligned}
$$

(Appendix A.2 provides details on this derivation.) In $\widehat{P}^{1,2}$, only two distances are known. Three values are possible for the third distance: 1, 2, or 3 (see top row of Fig. 1). Again using the extended Bethe approximation, while ignoring correlations
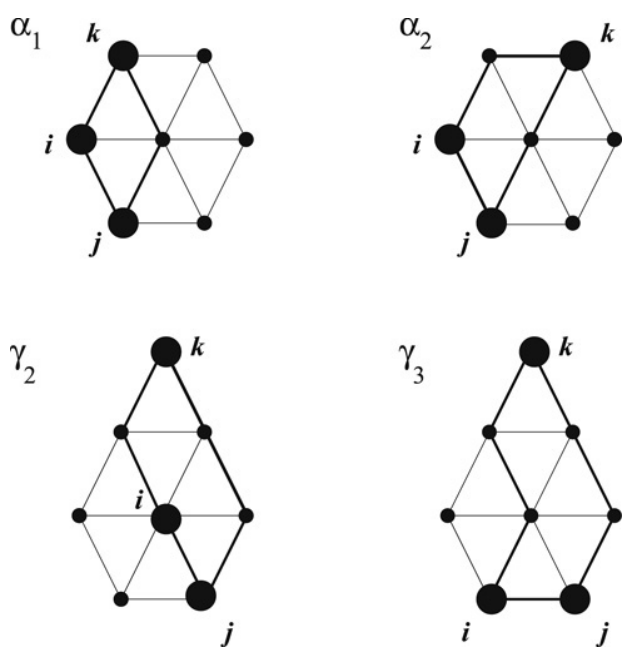

Fig. 1. Illustration on the triangular grid of configurations of triplets of nodes $i, j, k$ that fall into the categories measured by $\alpha_{1}$ and $\alpha_{2}$ (top row), and by $\gamma_{2}$ and $\gamma_{3}$ (bottom row). Enlarged nodes are part of the triplet, while thickened lines depict (not necessarily unique) paths between each two of the three nodes that have shortest length and thus define their distance.

at distance 3, we obtain

$$
\begin{aligned}
& \widehat{P}^{1,2}\left(z_{i}, z_{j}, z_{k}\right)=\frac{P^{1}\left(z_{i}, z_{j}\right) P^{2}\left(z_{j}, z_{k}\right)}{P\left(z_{i}\right) P\left(z_{j}\right) P\left(z_{k}\right)} \\
& \quad \times\left[\alpha_{1} P^{1}\left(z_{i}, z_{k}\right)+\alpha_{2} P^{2}\left(z_{i}, z_{k}\right)+\alpha_{3} P\left(z_{i}\right) P\left(z_{k}\right)\right] .
\end{aligned}
$$

The coefficients $\alpha_{1}, \alpha_{2}$, and $\alpha_{3}=1-\alpha_{1}-\alpha_{2}$ represent the proportions of the three corresponding triplet configurations. The extended closure therefore enables us to capture the influence of new geometrical characteristics of the graph that go beyond the clustering coefficient $\theta$ and which, according to the approximation derived here, must be expected to affect the dynamics systematically. We refer to $\alpha_{1}, \alpha_{2}$, and $\alpha_{3}$ as a graph's square clustering coefficients. Their influence can be explored based on the distance- 2 order- 2 closure derived here.

Finally, pair probabilities for states 00 and 11 are readily recovered from the singlet probability for state 1 and the pair probability for state 01 ,

$P^{1}(11)=\rho-P^{1}(01)$,
$P^{1}(00)=1-\rho-P^{1}(01)$.

The dynamic system given by Eqs. (2), (3) and (13) has thus been closed by the approximations in Eq. (12) (and the corresponding equation for $\left.\widehat{P}^{1,1}(101)\right),(14)-(16)$.

\subsection{Incorporating distance-3 correlations}

The method detailed above can be used to refine moment closure approximations by including correlations between nodes separated by more than two edges. We have implemented one more step in this scheme for successive refinement, by accounting for distance-3 correlations. It turns out that this enhancement modifies only the term with weight $\alpha_{3}$ in Eq. (15), as this is the only place where a distance larger than 2 needs to be considered between the three nodes involved in the triplet probabilities. 


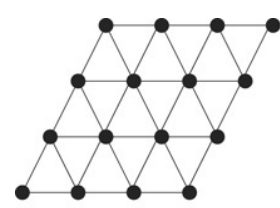

a

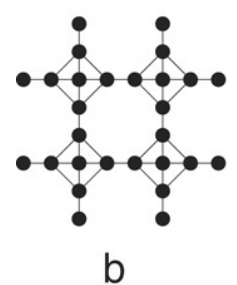

b

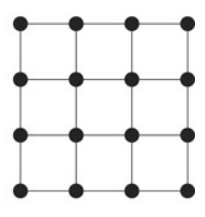

C

Fig. 2. The three studied graphs with homogeneous degree: (a) triangular grid with $h=6$, (b) 4-regular graph with $h=4$, (c) square grid with $h=4$.

Applying the same logic as introduced above for distance2 correlations, we derive this further extension through the following steps:

1. We add a fourth equation in the system, to describe the dynamics of $P^{3}(01)$.

2. We express the transition rates $T^{3}(01 \rightarrow 11)$ and $T^{3}(00 \rightarrow$ $01)$ in terms of the probabilities $P^{1,3}\left(z_{i}, z_{j}, z_{k}\right)$ that three nodes $i, j$, and $k$ are in states $z_{i}, z_{j}$, and $z_{k}$, given that $d_{i j}=1, d_{j k}=3$, and $d_{i k}$ is unknown (while $i$ is not on the path between $j$ and $k$ ).

3. We decompose the approximation of $P^{1,3}\left(z_{i}, z_{j}, z_{k}\right)$ considering three possibilities for $d_{i k}: 2,3$, or 4 (see bottom row of Fig. 1). The case $d_{i k}=1$ does not occur, since it would imply $d_{j k}=2$. The proportions $\gamma_{2}, \gamma_{3}$, and $\gamma_{4}=$ $1-\gamma_{2}-\gamma_{3}$ of these three triplet configurations can be determined for any graph.

4. We apply the extended Bethe approximation in Eq. (10) for $d_{i k}=2,3$, and 4 while ignoring correlations at distance 4 , $P^{4}\left(z_{i}, z_{k}\right)=P\left(z_{i}\right) P\left(z_{k}\right)$.

By going one step further in the refinement of the moment closure approximation we can thus introduce, and study the influence of, the two new graph characteristics $\gamma_{2}$ and $\gamma_{3}$, which help characterize a graph's geometrical features beyond its clustering coefficient $\theta$ and its square clustering coefficients $\alpha_{1}$ and $\alpha_{2}$.

\section{Numerical results}

We derived the distance- 2 and distance- 3 extensions of the normalized Bethe approximation $(N B A)$, power-1 closure $(P 1)$, and power-2 closure $(P 2)$ according to the general method detailed for the Bethe approximation in the previous section. On this basis, we systematically compared the performance of the following six closures: mean-field approximation $(M F)$, pair approximation $(P A)$, Bethe approximation $(B A), N B A, P 1$, and $P 2$ without and, when applicable, with the extensions derived above for incorporating correlations at distances 2 and 3. The performance of closures was evaluated for the contact process, by estimating the transmission rate's critical
Table 1

Statistics characterizing geometrical features of the three studied graphs: $h$ is the degree; $\theta$ is the clustering coefficient; $\alpha_{1}, \alpha_{2}$, and $\alpha_{3}$ are the square clustering coefficients affecting distance- 2 closures, and $\gamma_{2}, \gamma_{3}$, and $\gamma_{4}$ are the coefficients affecting distance- 3 closures

\begin{tabular}{lcccccccc}
\hline Graph & $h$ & $\theta$ & $\alpha_{1}$ & $\alpha_{2}$ & $\alpha_{2}$ & $\gamma_{2}$ & $\gamma_{3}$ & $\gamma_{4}$ \\
\hline $\begin{array}{l}\text { Triangular } \\
\text { grid }\end{array}$ & 6 & $2 / 5$ & $2 / 15$ & $6 / 15$ & $7 / 15$ & $2 / 15$ & $6 / 15$ & $7 / 15$ \\
$\begin{array}{l}\text { 4-regular } \\
\text { graph }\end{array}$ & 4 & $2 / 5$ & $4 / 45$ & $4 / 15$ & $29 / 45$ & $2 / 45$ & $12 / 45$ & $31 / 45$ \\
\begin{tabular}{l} 
Square grid \\
\hline
\end{tabular} & 4 & 0 & $2 / 9$ & 0 & $7 / 9$ & $2 / 5$ & 0 & $2 / 5$ \\
\hline
\end{tabular}

value $\beta_{c}$ and by examining the transients and equilibrium of the singlet probability $\rho$. Accurate predictions of $\beta_{c}$, and of equilibrium probabilities $\rho$ close to $\beta_{c}$, are known to be much more challenging than predictions of $\rho$ far away from $\beta_{c}$.

\subsection{Method of comparison}

Three different graphs with homogeneous degree were studied (Fig. 2). The first graph is conventionally referred to as the triangular grid $(h=6, \theta=0.4$; Fig. 2(a)). The second graph is the 4-regular graph $(h=4, \theta=0.4$; Fig. 2(b)), which shares the clustering coefficient with the triangular grid, but differs with regard to the degree and the coefficients affecting distance- 2 and distance- 3 correlations. The third graph is the square grid $(h=4, \theta=0$; Fig. 2(c)), which shares a degree of 4 with the 4-regular graph, but is characterized by a vanishing clustering coefficient. All graph statistics necessary for implementing the closureapproximations are easily obtained by analytical combinatorial computation; the resultant values (assuming infinitely extended graphs) are reported in Table 1.

We used the Runge-Kutta integration method from the package odesolve of the scientific software environment $\mathrm{R}$ to solve the ODE systems resulting from the different moment closure approximations. Estimations of $\rho$ during transients and at equilibrium were obtained from simulations of the continuous-time contact process by averaging over 100 different realizations, each of which proceeded for 200,000 iterations. An iteration was defined as the modification of the state of a single node in the graph, according to the rates given by Eqs. (1a) and (1b). The time steps between successive iterations were drawn from the appropriate exponential distribution. We ran simulations of the contact process on graphs with about $N=5000$ nodes. Both the ODE models and the contact process simulations were initialized with a $20 \%$ proportion of infected nodes (allocated at random in the simulations).

\subsection{Comparison of different closures}

\subsubsection{Prediction of critical transmission rate}

Table 2 shows the simulation-based estimates of $\beta_{c}$, together with predictions resulting from the six moment closure approximations for the three studied graphs. Exact analytical results 
Table 2

Estimations of the critical value $\beta_{c}$ of the contact process transmission rate from simulations and predictions obtained by solving the ODE systems resulting from the six different moment closure approximation without (classical) and with extension to distance- 2 and distance- 3 correlations

\begin{tabular}{|c|c|c|c|}
\hline \multicolumn{4}{|l|}{ (a) } \\
\hline Closure & Classical & Distance-2 & Distance-3 \\
\hline Mean field & $1 / 4$ & & \\
\hline Pair approximation & $1 / 3$ & & \\
\hline Bethe approximation & 0.369 & 0.406 & 0.366 \\
\hline Normalized $B A$ & 0.371 & 0.413 & 0.424 \\
\hline Power-1 & 0.440 & - & - \\
\hline Power-2 & 0.295 & 0.319 & 0.334 \\
\hline \multicolumn{4}{|l|}{ (b) } \\
\hline Simulation & 0.400 & & \\
\hline Closure & Classical & Distance-2 & Distance-3 \\
\hline Mean field & $1 / 4$ & & \\
\hline Pair approximation & $1 / 3$ & & \\
\hline Bethe approximation & $1 / 3$ & 0.367 & 0.355 \\
\hline Normalized $B A$ & $1 / 3$ & 0.370 & 0.393 \\
\hline Power-1 & 0.33 & - & - \\
\hline Power-2 & 0.260 & 0.266 & 0.272 \\
\hline \multicolumn{4}{|l|}{ (c) } \\
\hline Simulation & 0.253 & & \\
\hline Closure & Classical & Distance-2 & Distance-3 \\
\hline Mean field & $1 / 6$ & & \\
\hline Pair approximation & $1 / 5$ & & \\
\hline Bethe approximation & 0.215 & 0.234 & 0.213 \\
\hline Normalized $B A$ & 0.215 & 0.236 & 0.243 \\
\hline Power-1 & 0.239 & - & - \\
\hline Power-2 & 0.174 & 0.177 & 0.180 \\
\hline
\end{tabular}

(a) 4-regular graph, (b) square grid, (c) triangular grid. No value is reported when a closure leads to a negative value for the singlet probability at equilibrium.

for $\beta_{c}$ are available for $M F$ and $P A(1 / h$ and $1 /(h-1)$, respectively). When $\theta=0$, classical $B A$ and $N B A$ are equal to $P A$.

Our results show that (1) with the exception of $P 1$, predictions based on distance-1 (classical) closures are poor, following the ordering $M F<P 2<P A \leq B A \leq N B A$, where $M 1<M 2$ means that method $M 1$ is less accurate than method $M 2$; (2) $P 1$ at distance 1 is more accurate for the 4regular graph than for the triangular grid; (3) accounting for correlations at distances 2 and 3 improves the predictions of $N B A$, whereas the improvement for $B A$ is restricted to distance 2 ; and (4) the distance- 2 and distance- 3 extensions destabilize $P 1$, because negative values for the triplet probabilities are produced. Fig. 3 illustrates results (2) and (3) around the values of $\beta_{c}$ estimated from simulations.

\subsubsection{Prediction of singlet probability during transients and at equilibrium}

Two approximations are retained for testing predictions of $\rho$ during transients and at equilibrium: $N B A$ with extension to distance-3 correlations and $P 1$ without extension. Fig. 4 shows the dynamics of the singlet probability $\rho$ for the 4-regular graph, based on simulations and on these two approximations.
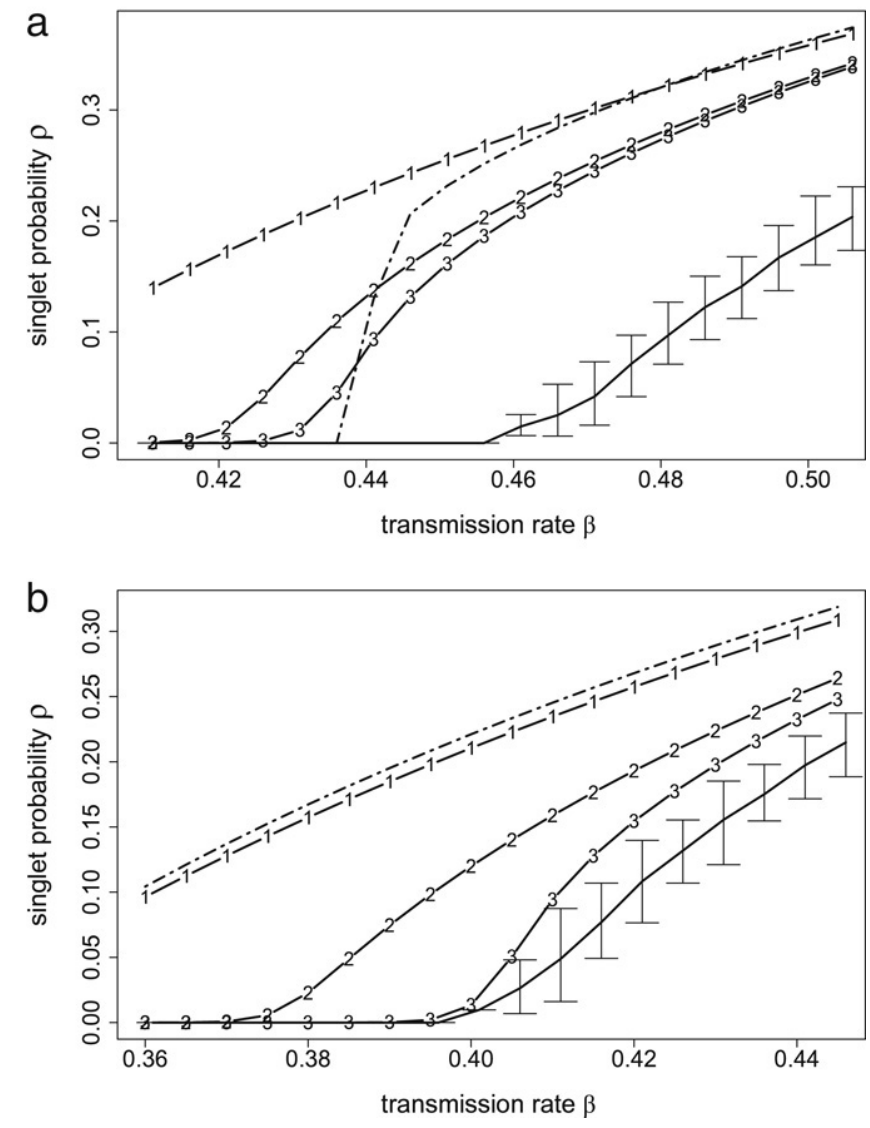

Fig. 3. Predictions of the singlet probability $\rho$ at equilibrium close to $\beta_{c}$ by $N B A$ with extension to longer-range correlations and by $P 1$ without extension. Continuous curves with error bars: mean and $90 \%$ confidence intervals from simulations; continuous curves with symbols 1 to 3 : NBA based on distance1 (classical), distance-2, and distance-3 correlations, respectively; dash-dotted curve: $P 1$ without extension to longer-range correlations. (a) 4-regular graph, (b) square grid.

No other approximation, without or with extension when applicable, resulted in better predictions (results not shown). Three cases can be distinguished:

1. For $\beta<\beta_{c}$ (Fig. 4(a)), the transients towards extinction are well predicted by $N B A$ with extension and by $P 1$ without extension (and with lesser accuracy by the other approximations).

2. For $\beta \simeq \beta_{c}$ with $\beta>\beta_{c}$ (Fig. 4(b)), all approximations overestimate the probability $\rho$, both at equilibrium and during the transients; however, this overestimation is smaller for $N B A$ with extension and for $P 1$ without extension than it is for the other approximations.

3. For $\beta \gg \beta_{c}$ (Fig. 4(c)), all methods correctly predict the probability $\rho$ at equilibrium, while the transients are predicted less accurately.

\subsection{Influence of network structure}

The extended closures we have introduced above can also be used to understand and predict the influence of those geometrical features of a graph that the closures have been shown to depend on. Owing to their key role in the resultant moment-based approximations, we propose these features as 

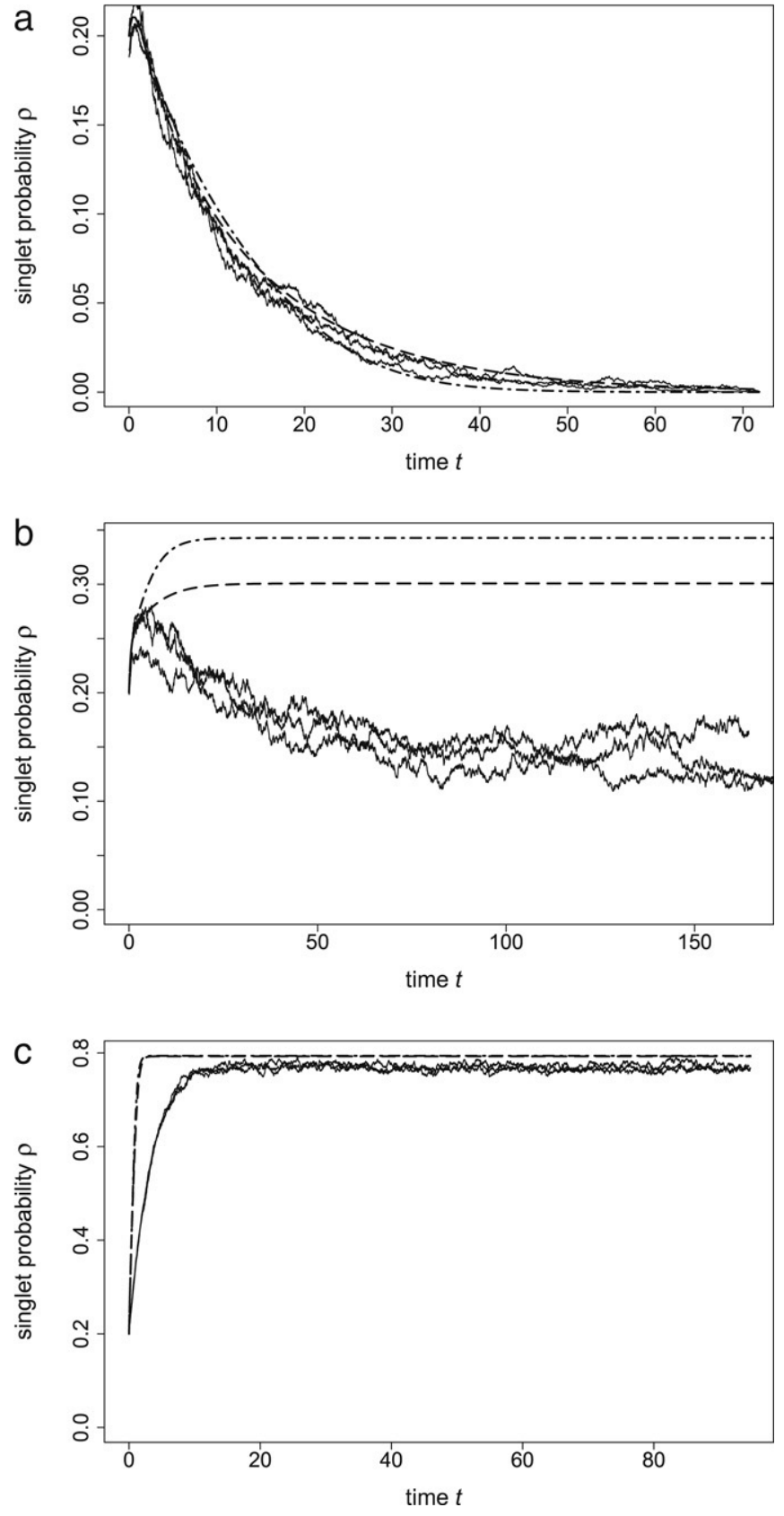

Fig. 4. Predictions of the singlet probability $\rho$ during transients for the 4 regular graph by $N B A$ with extension to longer-range correlations and by $P 1$ without extension. Continuous curves: three independent realizations of the contact process; dashed curve: NBA with extension to distance- 3 correlations; dash-dotted curve: $P 1$ without extension to longer-range correlations. (a) $\beta=$ 0.39 , (b) $\beta=0.49$, (c) $\beta=1.3$.

good candidates for characterizing a graph's dynamically relevant geometrical properties.

We tested the influence of the clustering coefficient $\theta$ and of the square clustering coefficients $\alpha_{1}, \alpha_{2}$, and $\alpha_{3}$ on the equilibrium probability $\rho$ using $N B A$ with extension to distance3 correlations. Whereas this closure and the classical $P 1$ provided the best approximations, the latter does not enable taking into account the square clustering coefficients. To test the influence of a given feature of the geometry $(\theta$, or the vector $\left(\alpha_{1}, \alpha_{2}, \alpha_{3}\right)$, with $\left.\alpha_{1}+\alpha_{2}+\alpha_{3}=1\right)$, we solved the ODE system

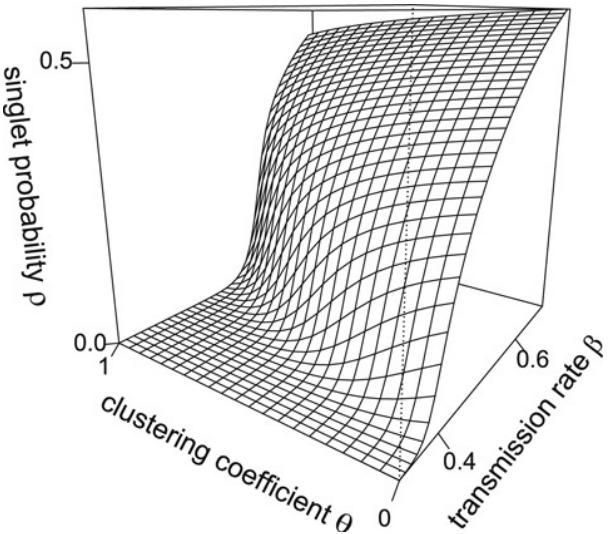

Fig. 5. Predictions of the influence of the clustering coefficient $\theta$ on the singlet probability $\rho$ at equilibrium for any graph with homogeneous degree $h=4$ and different transmission rates $\beta$.

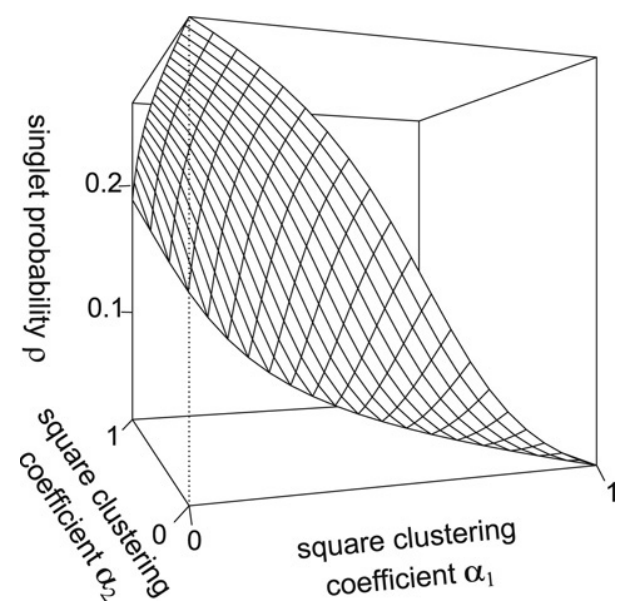

Fig. 6. Predictions of the influence of the square clustering coefficients $\alpha_{1}$ and $\alpha_{2}$ on the singlet probability $\rho$ at equilibrium for any graph with homogeneous degree $h=4$ and a transmission rate $\beta=0.47$.

of the moment-based approximation for a range of values of the corresponding parameter, while leaving the other parameters unchanged.

Fig. 5 shows the influence of the clustering coefficient $\theta$ for different values of the transmission rate $\beta$. We see that the role of $\theta$ is highly dependent on $\beta$ : for high values of $\beta$ (implying a high probability $\rho$ of infected nodes at equilibrium) there is little effect of $\theta$. This is consistent with the acceptable predictive accuracy of the mean-field approximation (which completely ignores the graph's geometry) for large $\beta$. The effect of $\theta$ increases as $\beta$ decreases towards its critical value. We recover the fact that a high clustering coefficient $\theta$ impedes disease propagation (Keeling, 1999; Proulx et al., 2005). This is because infected nodes in a highly clustered region of the interaction network share many nearest neighbours; these links are then wasted by becoming redundant as paths for disease propagation.

Fig. 6 shows the influence of the square clustering coefficients $\alpha_{1}, \alpha_{2}$ and $\alpha_{3}$. We see that the partitioning of triplet configurations as measured by these coefficients can significantly modify the equilibrium probability $\rho$. Their effect is especially strong when the transmission rate $\beta>\beta_{c}$ is 
relatively low. Specifically, when $\alpha_{1}$ is dominant among the three square clustering coefficients, $\rho$ is lowest, whereas when $\alpha_{3}$ is dominant, $\rho$ is highest. The interpretation of this result is analogous to that for $\theta$ : a high value of $\alpha_{1}$ implies a redundancy of paths of length 2 between pairs of nodes, so that infected nodes have more overlapping contacts, which consequently are wasted from the perspective of disease propagation. Again, the sensitivity to these geometric features decreases as the transmission rate $\beta$ increases and vanishes altogether for very high values of $\beta$.

\section{Discussion}

Stochastic dynamical systems on graphs provide powerful tools for understanding and quantifying the role of spatial or social structures, and of the resultant contact networks, in the emergence and spread of infectious diseases. Simulations of such dynamics are computationally intensive and analytical results are rarely available. A standard method of investigation is thus the derivation of approximations based on order2 moment closures. Here we introduced a new method for improving classical order-2 moment closure approximations by accounting for longer-range correlations, thus revealing the importance of previously unrecognized general geometric features of contact networks.

Longer-range correlations are particularly important in models of population dynamics in continuous space, defined through spatial kernels for local processes such as competition and movement, rather than through nearest-neighbour interaction. It is therefore not surprising that longer-range correlations have more naturally been taken into account in those models (Bolker and Pacala, 1997; Murrel et al., 2004). However, to our knowledge, the importance of longer-range correlations for the quality of moment closures has been little studied in discrete space up until now. While methods have been developed for improving classical order-2 closures on graphs, in order to compensate for neglected correlations (Sato et al., 1994; Snyder and Nisbet, 2000; Filipe and Gibson, 2001), these methods rely on the introduction of new parameters estimated through simulations or heuristic choices. By contrast, the approach we propose here is self-contained and the new parameters appearing in the ODEs are clearly identified as geometric characteristics of the graph.

In analyzing the performance of our new method for moment closures, we have focused on the contact process. This simple model of disease dynamics involves as a single essential parameter a disease's transmission rate $\beta$ between neighbouring hosts. And yet this model's behaviour is difficult to predict accurately, especially in the vicinity of the critical value $\beta_{c}$ below which the disease dies out. For these reasons the contact process serves as a valuable benchmark for the performance of moment closure approximations.

We have systematically evaluated the effect of the choice of moment closure on the quality of the resultant predictions. We compared five different forms of order- 2 closure, several of them introduced from studies in continuous space and extended here to discrete space. Whereas other variations of the classical pair approximation have been developed, we could not consider all of them, since either they cannot be used to account for longer-range correlations (Sato et al., 1994; Filipe and Gibson, 2001; Bauch, 2005) or they are very similar to the approximations we have studied here (e.g. Van Baalen, 2000 or Keeling, 1999), have proposed forms close to the Bethe approximation, taking into account the clustering coefficient). For all the moment closures we have considered and for which this was feasible, we derived the corresponding extensions based on accounting for longer-range correlations.

We found that, (1) for $\beta \simeq \beta_{c}$ with $\beta<\beta_{c}$, transients towards extinction are often poorly predicted by classical moment closure approximations, while introducing longerrange correlations improves the quality of the approximation, (2) for $\beta \simeq \beta_{c}$ with $\beta>\beta_{c}$, all considered approximations overestimate the disease prevalence both at equilibrium and during transients, and (3) for $\beta \gg \beta_{c}$, all considered approximations predict disease prevalence at equilibrium with good accuracy but perform less well in predicting the transients. Such a dependence in the performance of moment closures on different parameter regimes (subcritical, critical, and metastable) has already been pointed out (e.g. Krishnarajah et al., 2005).

Our systematic comparisons then demonstrated that the two moment closures leading to the best fit for disease prevalence, both at equilibrium and during transients, are the power-1 closure without extension and the normalized Bethe approximation with distance- 3 correlations. Even though these two closures do not always predict the critical value of transmission rate with high accuracy, they provide reasonable estimations. If the main objective is to explore the influence of a graph's characteristics, rather than to obtain precise quantitative predictions, the normalized Bethe approximation with distance3 correlations can be relied upon for good results.

Our study has also demonstrated that the length of loops in a contact graph may offer a valuable lead for selecting the most suitable moment closure. It is well known from statistical physics and from the study of Bayesian networks that dynamics on graphs without loops, commonly known as trees, are far simpler to analyse. Several approximation methods are rigorous on trees, but still work well on graphs with loops (e.g. Yedidia et al., 2005). On regular graphs with homogeneous degree, as those studied here, loops can be triangles, squares, or may be longer. While some approximations that do not account for long-range correlations provide good predictions when loops are short (resulting in a large clustering coefficient, such as for the triangular grid), the new method proposed here, by adding information about longer-range correlations, significantly improves the estimation of the critical transmission rate $\beta_{c}$ when loops are longer than triangles. The improvements are particularly striking when minimal loops are squares (so that the clustering coefficient vanishes, such as for the square grid).

Based on these considerations, we offer the following suggestions for selecting suitable moment closures for approximating dynamics in discrete space: (1) mean-field approximation when studying random graphs far from the critical point or graphs with high mean degree; (2) pair 
approximation when predictions are needed closer to a critical point for graphs without loops (trees); (3) classical order-2 closures that distinguish between open and closed triplets when dynamics are studied close to a critical point and the graph's clustering coefficient is intermediate to high; (4) normalized Bethe approximation extended to account for longer-range correlations when studying dynamics close to a critical point for graphs that have small clustering coefficients and exhibit longer loops. In particular, it should be appreciated that our new method yields improvements under conditions that are known to cause the mean-field approximation's failure: our method offers satisfactory predictions close to critical points and for graphs with low homogeneous degree.

Our derivation of extensions of classical closures enables us to draw attention to several new geometrical characteristics of graphs. We have shown that these are important for simplifying the spatiotemporal complexity of dynamics on graphs in general and for studying the structural determinants of disease spread in particular. In addition to the abovementioned clustering coefficient, we have identified several new coefficients: the square clustering coefficients affecting distance- 2 correlations, as well as further clustering coefficients affecting distance-3 correlations. In Caldarelli et al. (2004), the authors pointed out that the clustering coefficient alone may not suffice for understanding the key features of contact networks and therefore considered extensions based on more complex patterns of nodes. Here we have formally linked such descriptions with the spatiotemporal dynamics of the contact process. Exploring the dynamic implications of clustering and square clustering coefficients is easy based on the extended normalized Bethe approximation, and yields results that are valid for whole classes of graphs sharing given combinations of these coefficients. In this way we have found that the influence of these coefficients, and thus of the corresponding features of graph geometry, is strong for low to intermediate values of transmission rate, as these coefficients serve as indicators of path redundancy in a graph. For graphs with homogeneous degree, analytical computation of these coefficients is straightforward. When considering more complex contact networks, appropriate algorithms are readily available for evaluating such coefficients (Schreiber and Schwöbbermeyer, 2005, and references therein).

While this study has focused on graphs with homogeneous degree, real interaction networks (except in some particular cases such as orchards) tend to be complex (which includes social contact networks, networks of airline routes, and ecological networks; Newman, 2003 and Proulx et al., 2005) and are characterized by more general degree distributions. The methodology presented here can be extended without conceptual problems to these situations and could thus help account for geometrical features specific to nonhomogeneous graphs. Pair approximation and Bethe approximations have already been derived for a graph with a general degree distribution (Peyrard and Franc, 2005). Combining the influence of degree distributions and of long-range correlations in a unified framework can be expected to provide increasingly accurate moment closure approximations of real spatiotemporal dynamics even close to critical points.

\section{Acknowledgments}

The authors are indebted to the anonymous referees for their valuable suggestions and helpful comments on a previous version of this manuscript.

\section{Appendix}

\section{A.1. Dynamics of $P^{1}(01)$}

Pairs of nodes at distance 1 in state 01 can originate from a node in a 00-pair becoming infected or from a node in a 11-pair becoming susceptible. 01-pairs can disappear either by infection of the susceptible node or by recovery of the infected one. Denoting by $T^{1}\left(z_{1}, z_{2} \rightarrow z_{3}, z_{4}\right)$ the transition rate of a pair of nodes at distance 1 from state $\left(z_{1}, z_{2}\right)$ to state $\left(z_{3}, z_{4}\right)$, these considerations yield the dynamics of $P^{1}(01)$,

$$
\begin{aligned}
\frac{d P^{1}(01)}{d t}= & P^{1}(00) T^{1}(00 \rightarrow 01)+P^{1}(11) T^{1}(11 \rightarrow 01) \\
& -P^{1}(01) T^{1}(01 \rightarrow 11)-P^{1}(01) T^{1}(01 \rightarrow 00) .
\end{aligned}
$$

The transition rates $T^{1}(11 \rightarrow 01)$ and $T^{1}(01 \rightarrow 00)$ are equal to 1 , while the transition rates $T^{1}(00 \rightarrow 01)$ and $T^{1}(01 \rightarrow 11)$ depend on the state of the neighbourhood of the node moving from state 0 to state 1 .

To see this, let us consider $T^{1}(01 \rightarrow 11)$ and introduce some suitable notations. We will refer to as $i$ the node changing from 0 to 1 and as $j$ the node staying in state 1 . With $N(i)$ representing the set of the $h$ nodes linked with $i$ by an edge, and with $z_{N(i)}^{t}$ denoting the state of the nodes in the neighbourhood of $i$ at time $t$, then the number of neighbours of node $i$ that are infected at time $t$ is $a_{i}\left(z_{N(i)}^{t}\right)=\sum_{l \in N(i)} \delta_{1, z_{l}^{t}}$, with $\delta_{1,0}=0$ and $\delta_{1,1}=1$. Since the state of node $j$ is known, we can rewrite this expression as $1+\sum_{l \in N(i) \backslash\{j\}} \delta_{1, z_{l}^{t}}$. Using properties of the mathematical expectation $E$, we thus obtain

$$
\begin{aligned}
T^{1}(01 \rightarrow 11) & =E\left[\beta a_{i}\left(z_{N(i)}^{t}\right) \mid z_{i}^{t}=0, z_{j}^{t}=1\right] \\
& =\beta\left(1+\sum_{l \in N(i) \backslash\{j\}} E\left[\delta_{1, z_{l}^{t}} \mid z_{i}^{t}=0, z_{j}^{t}=1\right]\right) \\
& =\beta\left(1+\sum_{l \in N(i) \backslash\{j\}} P\left(z_{l}^{t}=1 \mid z_{i}^{t}=0, z_{j}^{t}=1\right)\right) \\
& =\beta\left(1+(h-1) \frac{P^{1,1}(101)}{P^{1}(01)}\right) .
\end{aligned}
$$

The same logic leads to

$$
T^{1}(00 \rightarrow 01)=\beta(h-1) \frac{P^{1,1}(100)}{P^{1}(00)} .
$$

With these results, the dynamics of $P^{1}(01)$ is given by

$$
\begin{aligned}
\frac{d P^{1}(01)}{d t}= & P^{1}(11)+\beta(h-1) P^{1,1}(100) \\
& -P^{1}(01)\left[1+\beta+\beta(h-1) \frac{P^{1,1}(101)}{P^{1}(01)}\right] .
\end{aligned}
$$




\section{A.2. Dynamics of $P^{2}(01)$}

To determine the dynamics of $P^{2}(01)$, we first consider the transition rate $T^{2}(01 \rightarrow 11)$ and specify that the node in the 01-pair that changes from 0 to 1 is called $j$ and the other node is called $k$. To determine this rate, we have to evaluate the probability for each neighbour of $j$ to be infected. One of these neighbours is the node in between $j$ and $k$ (since we know that the shortest path between $j$ and $k$ is of length 2). The ratio $\widehat{P}^{1,1,2}(011) / P^{2}(01)$ approximates the probability of this intermediate node to be infected, while $\widehat{P}^{1,2}(101) / P^{2}(01)$ approximates the probability of the $h-1$ other neighbours of $j$ to be infected, so that

$\hat{T}^{2}(01 \rightarrow 11)=\beta(h-1) \frac{\widehat{P}^{1,2}(101)}{P^{2}(01)}+\beta \frac{\widehat{P}^{1,1,2}(011)}{P^{2}(01)}$.

The triplet probability $\widehat{P}^{1,1,2}(011)$ is obtained using Eq. (10). To determine $\widehat{P}^{1,2}(101)$, we decompose $\widehat{P}^{1,2}\left(z_{i}, z_{j}, z_{k}\right)$ with respect to the distance between node $i$ and node $k$, in the same way we decomposed $\widehat{P}^{1,1}\left(z_{i}, z_{j}, z_{k}\right)$ using the clustering coefficient $\theta$. For this we recall that $P^{1,2}\left(z_{i}, z_{j}, z_{k}\right)$ is the probability to find three arbitrary nodes $i, j$, and $k$ in configuration $\left(z_{i}, z_{j}, z_{k}\right)$, given that the distance between $i$ and $j$ is 1 and that the shortest path between $j$ and $k$ is of length 2 . The nodes $i$ and $k$ may be at distance 1 (Fig. 1(a)), distance 2 (Fig. 1(b)), or distance 3. A distance of 4 is not possible because of the triangular inequality $d_{i k} \leq d_{i j}+d_{j k}=1+2=3$. As in Section 4.2, we denote by the square clustering coefficients $\alpha_{1}$, $\alpha_{2}$, and $\alpha_{3}$, respectively, the proportions of these three triplet configurations, so that we can decompose as follows,

$$
\begin{aligned}
\widehat{P}^{1,2}\left(z_{i}, z_{j}, z_{k}\right)= & \alpha_{1} \widehat{P}^{1,2,1}\left(z_{i}, z_{j}, z_{k}\right)+\alpha_{2} \widehat{P}^{1,2,2}\left(z_{i}, z_{j}, z_{k}\right) \\
& +\alpha_{3} \widehat{P}^{1,2,3}\left(z_{i}, z_{j}, z_{k}\right) .
\end{aligned}
$$

Finally, we apply the distance-2 Bethe closure to determine the three approximate triplet probabilities above,

$$
\begin{aligned}
\widehat{P}^{1,2}\left(z_{i}, z_{j}, z_{k}\right)= & \alpha_{1} \frac{P^{1}\left(z_{i}, z_{j}\right) P^{2}\left(z_{j}, z_{k}\right) P^{1}\left(z_{i}, z_{k}\right)}{P\left(z_{i}\right) P\left(z_{j}\right) P\left(z_{k}\right)} \\
& +\alpha_{2} \frac{P^{1}\left(z_{i}, z_{j}\right) P^{2}\left(z_{j}, z_{k}\right) P^{2}\left(z_{i}, z_{k}\right)}{P\left(z_{i}\right) P\left(z_{j}\right) P\left(z_{k}\right)} \\
& +\alpha_{3} \frac{P^{1}\left(z_{i}, z_{j}\right) P^{2}\left(z_{j}, z_{k}\right)}{P\left(z_{j}\right)}
\end{aligned}
$$

where in the third term we have assumed that the states of the pair of nodes at distance 3 are independent.

\section{References}

Albert, R., Barabási, A.-L., 2002. Statistical mechanics of complex networks. Rev. Modern Phys. 74, 47-97.

Bauch, C.T., 2005. The spread of infectious diseases in spatially structured populations: An invasory pair approximation. Math. Biosci. 198, 217-237.

Bolker, B.M., Pacala, S.W., 1997. Using moment equations to understand stochastically driven spatial pattern formation in ecological systems. Theor. Popul. Biol. 52, 179-197.

Caldarelli, G., Pastor-Satorras, R., Vespignani, A., 2004. Structure of cycles and local ordering in complex networks. Eur. Phys. J. B 38, 183-186.
Colizza, V., Barrat, A., Barthélemy, M., Vespignani, A., 2006. The modeling of global epidemics: Stochastic dynamics and predictability. Bull. Math. Biol. 68, 1893-1921.

Dickman, R., Martins de Oliveira, M., 2005. Quasi-stationary simulation of the contact process. Physica A 357, 134-141.

Dieckmann, U., Law, R., 2000. Relaxation projections and the method of moments. In: Dieckmann, U., Law, R., Metz, J.A.J. (Eds.), The Geometry of Ecological Interactions: Simplifying Spatial Complexity. Cambridge University Press, pp. 412-455 (Chapter 21).

Dieckmann, U., Law, R., Metz, J.A.J. (Eds.), 2000. The Geometry of Ecological Interactions: Simplifying Spatial Complexity. Cambridge Studies in Adaptive Dynamics. Cambridge University Press, UK.

Drossel, B., Schwabl, F., 1992. Self-organized critical forest-fire model. Phys. Rev. Lett. 69, 1629-1632.

Durrett, R., Levin, S., 1994. The importance of being discrete (and spatial). Theor. Pop. Biol. 46, 363-394.

Eguiluz, V., Klemm, K., 2002. Epidemic threshold in structured scale-free networks. Phys. Rev. Lett. 89, 108701.

Filipe, J.A.N., Gibson, G.J., 1998. Studying and approximating spatio-temporal models for epidemic spread and control. Philos. Trans. R. Soc. Lond. 353, 2153-2162.

Filipe, J.A.N., Gibson, G.J., 2001. Comparing approximations to spatiotemporal models for epidemics with local spread. Bull. Math. Biol. 63, 603-624.

Franc, A., 2004. Metapopulation dynamics as a contact process on a graph. Ecol. Comp. 1, 49-63.

Harris, T.E., 1974. Contact interactions on a lattice. Ann. Probab. 2, 969-988.

Iwasa, Y., 2000. Lattice models and pair approximation in ecology. In: Dieckmann, U., Law, R., Metz, J.A.J. (Eds.), The Geometry of Ecological Interactions: Simplifying Spatial Complexity. Cambridge University Press, pp. 227-251 (Chapter 13).

Keeling, M., 1999. The effects of local spatial structure on epidemiological invasions. Proc. R. Soc. Lond. B 266, 859-867.

Keeling, M., Woolhouse, M., May, R., Davies, G., Grenfell, B., 2003. Modelling vaccination strategies against foot-and-mouth disease. Nature 421, 136-142.

Keeling, M., 2005. The implications of network structure for epidemic dynamics. Theor. Pop. Biol. 67, 1-8.

Kikuchi, R., 1951. A theory of cooperative phenomena. Phys. Rev. 81, 988-1003.

Krishnarajah, I., Cook, A., Marion, G., Gibson, G., 2005. Novel moment closure approximations in stochastic epidemics. Bull. Math. Biol. 67, $855-873$.

Lavis, D.A., Bell, G.M., 1999. Statistical Mechanics of Lattice Systems: 1 Closed-Form and Exact Solutions. Springer Verlag.

Liggett, T.M., 1985. Interacting Particle Systems. Springer, New York.

Law, R., Dieckmann, U., 2000. A dynamical system for neighborhoods in plant communities. Ecology 81, 2137-2148.

Law, R., Murrell, D.J., Dieckmann, U., 2003. Population growth in space and time: Spatial logistic equations. Ecology 84, 252-262.

Marro, J., Dickman, R., 1999. Nonequilibrium Phase Transitions in Lattice Models. Monographs and Texts in Statistical Physics. Cambridge University Press, UK.

Matsuda, H., Ogita, A., Sasaki, N., Sato, K., 1992. Statistical mechanics of population: The lattice Lotka-Volterra model. Progr. Theoret. Phys. 88, 1035-1049.

Morita, T., 1994. Formal structure of the cluster variation method. Prog. Theor. Phys. 115 (Suppl.), 27-39.

Murrel, D., Dieckmann, U., Law, R., 2004. On moment closures for population dynamics in continuous space. J. Theoret. Biol. 229, 421-432.

Newman, M.E.J., 2003. The structure and function of complex networks. SIAM Rev. 45, 167-256.

Pastor-Satorras, R., Vespignani, A., 2001. Epidemic spreading in scale-free networks. Phys. Rev. Lett. 86, 3200-3203.

Petermann, T., De Los Rios, P., 2004a. Cluster approximations for epidemic processes: A systematic description of correlations beyond the pair level. J. Theor. Biol. 229, 1-11.

Petermann, T., De Los Rios, P., 2004b. The role of clustering and gridlike ordering in epidemic spreading. Phys. Rev. E 69, 066116. 
Peyrard, N., Franc, A., 2005. Cluster variational approximations for a contact process living on a graph. Physica A 358, 575-592.

Proulx, S., Promislow, D., Phillips, P., 2005. Network thinking in ecology and evolution. Trends Ecol. Evol. 20, 345-353.

Sato, K., Iwasa, Y., 2000. Pair approximations for lattice-based ecologica models. In: Dieckmann, U., Law, R., Metz, J.A.J. (Eds.), The Geometry of Ecological Interactions: Simplifying Spatial Complexity. Cambridge University Press, pp. 341-358 (Chapter 18).

Sato, K., Matsuda, H., Sasaki, A., 1994. Pathogen invasion and host extinction in lattice structured populations. J. Math. Biol. 32, 251-268.

Schreiber, S., Schwöbbermeyer, H., 2005. Frequency concepts and pattern detection for the analysis of motifs in networks. Trans. Comput. Syst. Biol 3 (LNBI 3737), 89-104.
Singer, A., 2004. Maximum entropy formulation of the Kirkwood superposition approximation. J. Ch. Phys. 121, 3657-3666.

Snyder, R., Nisbet, R., 2000. Spatial structure and fluctuations in the contact process and related models. Bull. Math. Biol. 62, 959-975.

Van Baalen, M., 2000. Pair approximations for different spatial geometries. In: Dieckmann, U., Law, R., Metz, J.A.J. (Eds.), The Geometry of Ecological Interactions: Simplifying Spatial Complexity. Cambridge University Press, pp. 359-387 (Chapter 19).

Van Baalen, M., Rand, D., 1998. The unit of selection in viscous populations and the evolution of altruism. J. Theoret. Biol. 193, 631-648.

Yedidia, J., Freeman, W., Weiss, Y., 2005. Constructing free energy approximations and generalized belief propagation algorithms. IEEE Trans. Inform. Theory 51, 2282-2312. 